\title{
Real fixed points and singular values of family of func- tions arising from generating function of unified generalized Apostol-type polynomials
}

\author{
Mohammad Sajid \\ College of Engineering, Qassim University, Buraidah, Al-Qassim, Saudi Arabia.
}

\begin{abstract}
Our main objective is to study the real fixed points and singular values of a two-parameter family of transcendental meromorphic functions $\mathrm{g}_{\lambda, \mathrm{n}}(z)=\lambda_{\frac{z}{\left(\mathrm{~b}^{z}-1\right)^{n}}}, \lambda \in \mathbb{R} \backslash\{0\}, z \in \mathbb{C} \backslash\{0\}, \mathrm{n} \in \mathbb{N} \backslash\{1\}, \mathrm{b}>0, \mathrm{~b} \neq 1$ in the present paper which obtains from generating function of the unified generalized Apostol-type polynomials. The real fixed points of $g_{\lambda, n}(x), x \in \mathbb{R} \backslash\{0\}$ with their stability are found for $n$ odd and $n$ even. It is shown that $g_{\lambda, n}(z)$ has infinite number of singular values. Further, it is seen that some critical values of $g_{\lambda, n}(z)$ lie in the closure of the disk and other lie in the exterior of the disk with center at the origin.
\end{abstract}

Keywords: Real fixed points, critical values, singular values, meromorphic function.

2010 MSC: 30D30, 37C25, 58K05.

(C)2019 All rights reserved.

\section{Introduction}

Often the fixed points and singular values are much important and play crucial role in the study of the behavior of nonlinear dynamical systems. For one parameter families of functions, the real fixed points are studied in [8,9] and the singular values are shown in [10,11]. For two-parameter families, the real fixed points are described in [4,13] and the singular values are found in [13, 14]. Generally, the real and complex dynamics of functions are associated to the fixed points and singular values and have become an interesting research area, make worth studying partially due to applicability of these for describing properties of the Julia sets and Fatou sets $[1-3,5,16]$. Using the real fixed points, the real dynamics of functions are investigated in $[6,12,17]$ and the bifurcation as well as chaos in the real dynamics of a two-parameter family of functions arises from generating function of generalized Apostoltype polynomials are discussed in [15]. Some advanced results on transcendental dynamics can be seen in $[7,19]$ by applicability of fixed points and singular values.

A point $x$ is called a fixed point of function $f(x)$ if $f(x)=x$. A fixed point $x_{f}$ is said to be attracting, neutral (rationally or irrationally indifferent), or repelling if $\left|f^{\prime}\left(x_{f}\right)\right|<1,\left|f^{\prime}\left(x_{f}\right)\right|=1$, or $\left|f^{\prime}\left(x_{f}\right)\right|>1$, 
respectively. Let $f: \mathbb{C} \rightarrow \mathbb{C}$. A point $z^{*} \in \mathbb{C}$ is said to be a critical point of $f(z)$ if $f^{\prime}\left(z^{*}\right)=0$. The value $f\left(z^{*}\right)$ corresponding to a critical point $z^{*}$ is called a critical value of $f(z)$. A point $w \in \hat{\mathbb{C}}=\mathbb{C} \cup\{\infty\}$ is said to be an asymptotic value for $f(z)$, if there exists a continuous curve $\gamma:[0, \infty) \rightarrow \hat{\mathbb{C}}$ satisfying $\lim _{t \rightarrow \infty} \gamma(t)=\infty$ and $\lim _{t \rightarrow \infty} f(\gamma(t))=w$. A singular value of $f$ is defined to be either a critical value or an asymptotic value of $f$.

Let

$$
\mathcal{G}=\left\{g_{\lambda, n}(z)=\lambda \frac{z}{\left(b^{z}-1\right)^{n}}: \lambda \in \mathbb{R} \backslash\{0\}, z \in \mathbb{C} \backslash\{0\}, n \in \mathbb{N} \backslash\{1\}, b>0, b \neq 1\right\}
$$

be two-parameter family of transcendental meromorphic functions which is neither even nor odd. This family of generating function is a unification of three generalized Apostol-type polynomials (ApostolBernoulli, Apostol-Euler, and Apostol-Genocchi) of order $\alpha \in \mathbb{C}$ which is given as [18]

$$
\begin{aligned}
& \left(\frac{2^{\mu} z^{\nu}}{\lambda b^{z}+a^{z}}\right)^{\alpha} c^{x z}=\sum_{k=0}^{\infty} z_{k}^{(\alpha)}(x ; \lambda ; a, b, c ; \mu ; v) \frac{z^{k}}{k !}, \\
& \left(|z|<\left|\frac{\log (-\lambda)}{\log \left(\frac{b}{a}\right)}\right| ; a \in \mathbb{C} \backslash\{0\} ; b, c \in \mathbb{R}^{+} ; a \neq b ; \alpha, \lambda, \mu, v \in \mathbb{C}, 1^{\alpha}:=1\right) .
\end{aligned}
$$

Setting $x=0, \lambda=1, \mu=0, \alpha=n, v=\frac{1}{n}$ and $a^{z}=-1$, we have

$$
\frac{z}{\left(b^{z}-1\right)^{n}}=\sum_{k=0}^{\infty} z_{k}^{(n)}\left(0 ; 1 ; a, b, c, 0, \frac{1}{n}\right) \frac{z^{k}}{k !} ;|z|<\left|\frac{\log (-1)}{\log \left(\frac{b}{a}\right)}\right| .
$$

For $n=1$, the real fixed points and singular values of $\lambda_{b^{z}-1}$ are investigated in [8,9] and [11] respectively. Moreover, it is a generalized family of functions $\frac{z}{\left(e^{z}-1\right)^{n}}$ [13] on base $b$ and the study of these kinds of maps are scarily found in the literature since most of the work has performed up to now deals with polynomials and rational maps.

The present paper describes the real fixed points and singular values of $g_{\lambda, n} \in \mathcal{G}$. In Theorem 2.3 and Theorem 2.4, the real fixed points of $g_{\lambda, n} \in \mathcal{G}$ with their stability are described for $n$ odd and $n$ even, respectively. It is seen that the function $g_{\lambda, n} \in \mathcal{G}$ has infinite number of singular values in Theorem 3.1. It is found that, in Theorem 3.3, $g_{\lambda, n}^{\prime}(z)$ has no zeros in the right half plane for $b>1$ and in the left half plane for $0<b<1$. In Theorem 3.5, $g_{\lambda, n}(z)$ maps the left half plane for $b>1$ and in the right half plane for $0<\mathrm{b}<1$ in the closure and into the exterior of the disk with center at origin according to two different regions, respectively. Further, in Theorem 3.6, it is observed that some critical values of $g_{\lambda, n} \in \mathcal{G}$ lie in the closure and other lie into the exterior of the disk with center at origin.

\section{Real fixed points of $g_{\lambda, n} \in \mathcal{G}$ and their nature}

The real fixed points of the functions $f_{\lambda, n} \in \mathcal{G}$ and their stability are discussed in this section. The following lemma is needed in the sequel:

Lemma 2.1. Let $h(x)=1-\frac{n x \ln b}{1-b^{-x}}, x \in \mathbb{R}, n \in \mathbb{N} \backslash\{1\}, b>0, b \neq 1$.

(i) For $\mathrm{b}>1, \lim _{x \rightarrow-\infty} \mathrm{h}(\mathrm{x})=1, \mathrm{~h}(0)=1-\mathrm{n}, \lim _{\mathrm{x} \rightarrow+\infty} \mathrm{h}(\mathrm{x})=-\infty, \mathrm{h}\left(\mathrm{x}_{1}^{*}\right)=-1$ and $\mathrm{h}\left(\mathrm{x}_{1}^{* *}\right)=-1$, where $\mathrm{x}_{1}^{*}$ and $\mathrm{x}_{1}^{* *}$ (depending on $\mathrm{n}$ and $\left.\mathrm{b}\right)$ are the unique negative real roots of the equation $(2-\mathrm{nx} \ln \mathrm{b}) \mathrm{b}^{\mathrm{x}}-2=0$ for odd $\mathrm{n} \in \mathbb{N} \backslash\{1\}$ and even $\mathrm{n} \in \mathbb{N} \backslash\{1\}$, respectively.

(ii) For $0<\mathrm{b}<1, \lim _{x \rightarrow+\infty} \mathrm{h}(\mathrm{x})=1, \mathrm{~h}(0)=1-\mathrm{n}, \lim _{\mathrm{x} \rightarrow-\infty} \mathrm{h}(\mathrm{x})=-\infty, \mathrm{h}\left(\mathrm{x}_{2}^{*}\right)=-1$ and $\mathrm{h}\left(\mathrm{x}_{2}^{* *}\right)=-1$, where $\mathrm{x}_{2}^{*}$ and $\mathrm{x}_{2}^{* *}$ (depending on $\mathrm{n}$ and $\mathrm{b}$ ) are the unique positive real roots of the equation $(2-\mathrm{nx} \ln \mathrm{b}) \mathrm{b}^{\mathrm{x}}-$ $2=0$ for odd $n \in \mathbb{N} \backslash\{1\}$ and even $n \in \mathbb{N} \backslash\{1\}$, respectively.

Proof. For $b>1$, it is easily seen that the function $\frac{x}{1-b^{-x}}$ tends to $0, \frac{1}{\ln b}$, or $\infty$ along as $x \rightarrow-\infty, x \rightarrow 0$, 
or $x \rightarrow+\infty$, respectively. Then

$$
\begin{aligned}
\lim _{x \rightarrow-\infty} h(x) & =1-n \ln b \lim _{x \rightarrow-\infty} \frac{x}{1-b^{-x}}=1, \\
h(0) & =1-n \ln b \lim _{x \rightarrow 0} \frac{x}{1-b^{-x}}=1-n, \\
\lim _{x \rightarrow+\infty} h(x) & =1-n \ln b \lim _{x \rightarrow+\infty} \frac{x}{1-b^{-x}}=-\infty, \\
h\left(x_{1}^{*}\right) & =1-\frac{n x_{1}^{*} \ln b}{1-b^{-x_{1}^{*}}}=1-\frac{n x_{1}^{*} \ln b}{\frac{n x_{1}^{*}}{2} \ln b}=-1 \text { since } 1-b^{-x_{1}^{*}}=\frac{n x_{1}^{*}}{2} \ln b, \\
h\left(x_{1}^{* *}\right) & =1-\frac{n x_{1}^{* *} \ln b}{1-b^{-x_{1}^{* *}}}=1-\frac{n x_{1}^{* *} \ln b}{\frac{n x_{1}^{* *}}{2} \ln b}=-1 \text { since } 1-b^{-x_{1}^{* *}}=\frac{n x_{1}^{* *}}{2} \ln b .
\end{aligned}
$$

This completes the proof of (i). Similarly, we can obtain the proof of (ii). The graphs of the function $h(x)=1-\frac{n x \ln b}{1-b^{-x}}$ are given in Figure 1 (a) for $n=3$ and $b=2$ and Figure 1 (b) for $n=2$ and $b=0.5$.

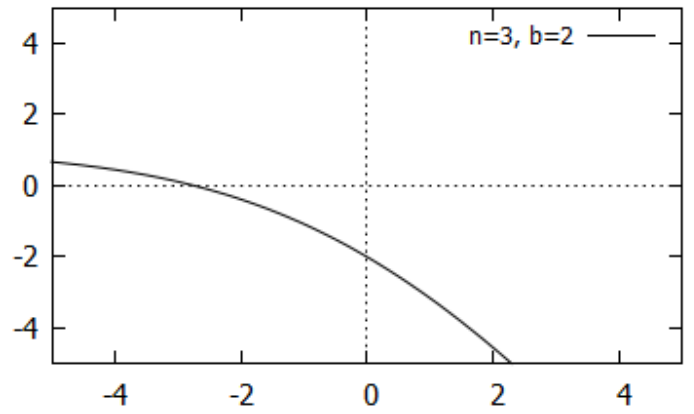

(a) For $n=3$ and $b=2$.

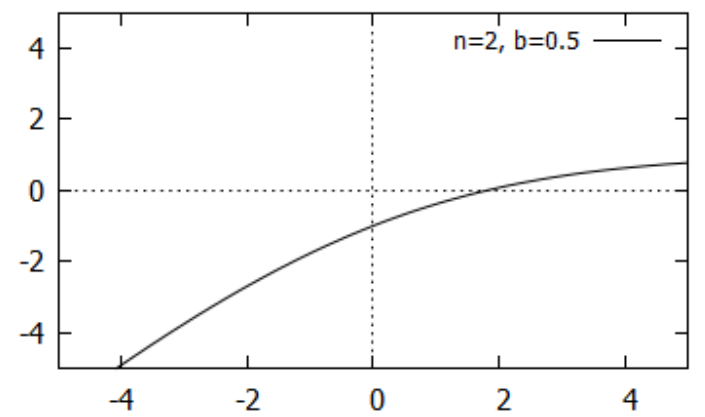

(b) For $n=2$ and $b=0.5$.

Figure 1: Graphs of $h(x)=1-\frac{n x \ln b}{1-b^{-x}}$.

Remark 2.2. It is easily observed that $x_{2}^{*}=-x_{1}^{*}$ and $x_{2}^{* *}=-x_{1}^{* *}$.

For $n$ odd, let us define

$$
\lambda^{*}=\frac{x^{*}}{g\left(x^{*}\right)}=\left(b^{x^{*}}-1\right)^{n}
$$

where $x^{*}$ is either $x_{1}^{*}$ or $x_{2}^{*}$ which is given in Lemma 2.1 for $b>1$ and $0<b<1$, respectively.

The following theorem describes the real fixed points of the function $g_{\lambda, n} \in \mathcal{G}$ and their nature for $n$ odd greater than 1.

Theorem 2.3. Let $\mathrm{g}_{\lambda, \mathrm{n}} \in \mathcal{G}$ for odd $\mathrm{n} \in \mathbb{N} \backslash\{1\}$.

(a) The function $\mathrm{g}_{\lambda, \mathrm{n}}(\mathrm{x})$ has a unique real fixed point $\mathrm{x}_{\lambda, \mathrm{n}}$ for $\lambda>-1$ and no real fixed point for $\lambda<-1$. The fixed point $x_{\lambda, n}$ of $g_{\lambda, n}(x)$ is

(i) negative if $-1<\lambda<0$ and positive if $\lambda>0$ for $\mathrm{b}>1$.

(ii) positive if $-1<\lambda<0$ and negative if $\lambda>0$ for $0<\mathrm{b}<1$.

(b) (i) If $\mathrm{b}>1$, the fixed point $x_{\lambda, \mathrm{n}}$ of $\mathrm{g}_{\lambda, \mathrm{n}}(\mathrm{x})$ is attracting for $-1<\lambda<\lambda^{*}$, rationally indifferent for $\lambda=\lambda^{*}$ and repelling for $\lambda^{*}<\lambda<0$ and $\lambda>0$.

(ii) If $0<\mathrm{b}<1$, the fixed point $x_{\lambda, n}$ of $\mathrm{g}_{\lambda, \mathrm{n}}(\mathrm{x})$ is repelling for $-1<\lambda<0$ and $0<\lambda<\lambda^{*}$, rationally indifferent for $\lambda=\lambda^{*}$ and attracting for $\lambda>\lambda^{*}$.

Proof. For fixed points of $g_{\lambda, n}(x)$, we have $\lambda \frac{x}{\left(b^{x}-1\right)^{n}}=x$.

(a) For $n$ odd greater than 1 , we get a unique fixed point $x_{\lambda, n}=\frac{\ln \left(1+\lambda^{1 / n}\right)}{\ln b}$. It follows that the fixed point $x_{\lambda, n}$ of $g_{\lambda, n}(x)$ is real for $\lambda>-1$ and no real fixed point for $\lambda<-1$. Therefore, the real fixed point $x_{\lambda, n}$ 
of $g_{\lambda, n}(x)$ is (i) positive if $\lambda>0$ and negative if $-1<\lambda<0$ for $b>1$; (ii) negative if $\lambda>0$ and positive if $-1<\lambda<0$ for $0<\mathrm{b}<1$.

(b) Since $x_{\lambda, n}$ is a fixed point of $g_{\lambda, n}(x)$, then

$$
g_{\lambda, n}^{\prime}\left(x_{\lambda, n}\right)=\lambda \frac{\left(1-n x_{\lambda, n} \ln b\right) b^{x_{\lambda, n}}-1}{\left(b^{x_{\lambda, n}}-1\right)^{n+1}}
$$

Substituting $\lambda=\left(b^{x_{\lambda, n}}-1\right)^{n}$ in equation (2.1), we get

$$
g_{\lambda, n}^{\prime}\left(x_{\lambda, n}\right)=\left(b^{x_{\lambda, n}}-1\right)^{n} \frac{\left(1-n x_{\lambda, n} \ln b\right) b^{x_{\lambda, n}}-1}{\left(b^{x_{\lambda, n}}-1\right)^{n+1}}=1-\frac{n x_{\lambda, n} \ln b}{1-b^{-x_{\lambda, n}}} .
$$

(i) For $b>1$ and $\lambda<0$, the fixed point $x_{\lambda, n}$ of $g_{\lambda, n}(x)$ is negative by part (a). It is seen that, using Lemma 2.1 (i), $-1<\mathrm{g}_{\lambda, \mathrm{n}}^{\prime}\left(\mathrm{x}_{\lambda, \mathrm{n}}\right)<1$ for $-1<\lambda<\lambda^{*}, \mathrm{~g}_{\lambda, \mathrm{n}}^{\prime}\left(\mathrm{x}_{\lambda, \mathrm{n}}\right)=-1$ for $\lambda=\lambda^{*}$ and $\mathrm{g}_{\lambda, \mathrm{n}}^{\prime}\left(\mathrm{x}_{\lambda, \mathrm{n}}\right)<-1$ for $\lambda^{*}<\lambda<0$. Hence, the fixed point $x_{\lambda, n}$ of $g_{\lambda, n}(x)$ is attracting for $-1<\lambda<\lambda^{*}$, rationally indifferent for $\lambda=\lambda^{*}$ and repelling for $\lambda^{*}<\lambda<0$.

For $\lambda>0$, the fixed point $x_{\lambda, n}$ of $g_{\lambda, n}(x)$ is positive by part (a). The function $\frac{n x \ln b}{1-b^{-x}}$ is positive and increasing from 0 to $\infty$ and the minimum value of the function $\frac{n x \ln b}{1-b^{-x}}$ is $n$ at $x=0$ in the interval $[0, \infty)$. For $n \geqslant 3$ and $x>0$, we can write $1-\frac{n \times \ln b}{1-b^{-x}}<1-n$. Hence, $\left|g_{\lambda, n}^{\prime}\left(x_{\lambda, n}\right)\right|=\left|1-\frac{n x_{\lambda, n} \ln b}{1-b^{-x_{\lambda, n}}}\right|>|1-n|>1$ since $n \geqslant 3$. Therefore, the fixed point $x_{\lambda, n}$ of $g_{\lambda, n}(x)$ is repelling for $\lambda>0$.

(ii) For $0<\mathrm{b}<1$, we construct the proof of this part similar as $\mathrm{b}>1$. For $-1<\lambda<0$, the fixed point $x_{\lambda, n}$ of $g_{\lambda, n}(x)$ is positive by part (a). The function $\frac{n x \ln b}{1-b^{-x}}$ is positive and decreasing from 0 to $\infty$ and the maximum value of the function $\frac{n x \ln b}{1-b^{-x}}$ is $n$ at $x=0$ in the interval $[0, \infty)$. For $n \geqslant 3$ and $x>0$, we can write $1-n<1-\frac{n \times \ln b}{1-b^{-x}}<1$. Hence, $1<\left|1-\frac{n x_{\lambda, n} \ln b}{1-b^{-x_{\lambda, n}}}\right|<|1-n|$. It follows that $1<\left|g_{\lambda, n}^{\prime}\left(x_{\lambda, n}\right)\right|<|1-n|$ since $n \geqslant 3$. Therefore, the fixed point $x_{\lambda, n}$ of $g_{\lambda, n}(x)$ is repelling for $-1<\lambda<0$.

For $\lambda>0$, by Lemma 2.1 (ii), we get $-1<g_{\lambda, n}^{\prime}\left(x_{\lambda, n}\right)<1$ for $\lambda>\lambda^{*}, g_{\lambda, n}^{\prime}\left(x_{\lambda, n}\right)=-1$ for $\lambda=\lambda^{*}$ and $g_{\lambda, n}^{\prime}\left(x_{\lambda, n}\right)<-1$ for $0<\lambda<\lambda^{*}$. Therefore, the fixed point $x_{\lambda, n}$ of $g_{\lambda, n}(x)$ is repelling for $0<\lambda<\lambda^{*}$, rationally indifferent for $\lambda=\lambda^{*}$ and attracting for $\lambda>\lambda^{*}$. This completes the proof of theorem.

For $n$ even, let us define

$$
\lambda^{* *}=\frac{x^{* *}}{g\left(x^{* *}\right)}=\left(b^{x^{* *}}-1\right)^{n}
$$

where $x^{* *}$ is either $x_{1}^{* *}$ or $x_{2}^{* *}$, which is given in Lemma 2.1 for $b>1$ and $0<b<1$, respectively.

The description of the real fixed points of $g_{\lambda, n} \in \mathcal{G}$ for $n$ even greater than 0 are different than for $n$ odd greater than 1 . The following theorem shows the real fixed points of the function $g_{\lambda, n} \in \mathcal{G}$ and their nature for $n$ even greater than 0 .

Theorem 2.4. Let $\mathrm{g}_{\lambda, \mathrm{n}} \in \mathcal{G}$ for even $\mathrm{n} \in \mathbb{N} \backslash\{1\}$.

(i) The function $\mathrm{g}_{\lambda, \mathrm{n}}(\mathrm{x})$ has two real fixed points for $0<\lambda<1$, a unique real fixed point for $\lambda \geqslant 1$ and no real fixed point for $\lambda<0$. If $\mathrm{b}>1$, one fixed point $x_{\lambda, \mathrm{n}}^{-}$is negative and other fixed point $x_{\lambda, \mathrm{n}}^{+}$is positive for $0<\lambda<1$; and only one fixed point $x_{\lambda, n}$ is positive for $\lambda \geqslant 1$. If $0<\mathrm{b}<1$, vice versa.

(ii) For $\mathrm{b}>1$, the fixed point $x_{\lambda, n}^{-}$is repelling for $0<\lambda<\lambda^{* *}$, rationally indifferent for $\lambda=\lambda^{* *}$ and attracting for $\lambda^{* *}<\lambda<1$. The fixed point $x_{\lambda, \mathrm{n}}^{+}$is repelling for $0<\lambda<1$ and the fixed point $x_{\lambda, \mathrm{n}}$ is repelling for $\lambda \geqslant 1$. For $0<\mathrm{b}<1$, the fixed point $x_{\lambda, n}^{+}$is repelling for $0<\lambda<\lambda^{* *}$, rationally indifferent for $\lambda=\lambda^{* *}$ and attracting for $\lambda^{* *}<\lambda<1$. The fixed point $x_{\lambda, \mathrm{n}}^{-}$is repelling for $0<\lambda<1$ and the fixed point $x_{\lambda, \mathrm{n}}$ is repelling for $\lambda \geqslant 1$.

Proof. For fixed points of $g_{\lambda, n}(x)$, we have $\lambda \frac{x}{\left(b^{x}-1\right)^{n}}=x$.

(i) For even $n \in \mathbb{N} \backslash\{1\}$, the fixed points are given as $x_{\lambda, n}=\frac{\ln \left(1 \pm \lambda^{1 / n}\right)}{\ln b}$. It follows that $g_{\lambda, n}(x)$ has two real fixed points for $0<\lambda<1$, a unique real fixed point for $\lambda \geqslant 1$ and no real fixed point for $\lambda<0$. For 
$0<\lambda<1$, it is easily seen that one fixed point $x_{\lambda, n}^{-}$is negative and other fixed point $x_{\lambda, n}^{+}$is positive for $\mathrm{b}>1$ and vice versa for $0<\mathrm{b}<1$. For $\lambda \geqslant 1, g_{\lambda, n}(x)$ has positive real fixed point $x_{\lambda, n}^{+}$for $b>1$ and has negative real fixed point $x_{\lambda, n}^{-}$for $0<b<1$.

(ii) Since $x_{\lambda, n}$ is a fixed point of $g_{\lambda, n}(x)$ and $\lambda=\left(b^{x_{\lambda, n}}-1\right)^{n}$, then we have

$$
g_{\lambda, n}^{\prime}\left(x_{\lambda, n}\right)=\left(b^{x_{\lambda, n}}-1\right)^{n} \frac{\left(1-n x_{\lambda, n} \ln b\right) b^{x_{\lambda, n}}-1}{\left(b^{x_{\lambda, n}}-1\right)^{n+1}}=1-\frac{n x_{\lambda, n} \ln b}{1-b^{-x_{\lambda, n}}} .
$$

For $\mathrm{b}>1$, by Lemma 2.1 (i), it is easily found that $\mathrm{g}_{\lambda, \mathrm{n}}^{\prime}\left(x_{\lambda, \mathrm{n}}^{-}\right)<-1$ for $0<\lambda<\lambda^{* *}, \mathrm{~g}_{\lambda, \mathrm{n}}^{\prime}\left(\mathrm{x}_{\lambda, \mathrm{n}}^{-}\right)=-1$ for $\lambda=\lambda^{* *}$ and $-1<g_{\lambda, n}^{\prime}\left(x_{\lambda, n}^{-}\right)<1$ for $\lambda^{* *}<\lambda<1$. It gives that the fixed point $x_{\lambda, n}^{-}$is repelling for $0<\lambda<\lambda^{* *}$, rationally indifferent for $\lambda=\lambda^{* *}$ and attracting for $\lambda^{* *}<\lambda<1$.

Now, with similar arguments as used for $n$ odd, we can deduce that the fixed point $x_{\lambda, n}^{+}$of $g_{\lambda, n}(x)$ is repelling for $0<\lambda<1$ and the fixed point $x_{\lambda, n}$ is repelling for $\lambda \geqslant 1$.

For $0<\mathrm{b}<1$, the proof of this part can be obtained similar as $\mathrm{b}>1$. The proof of the theorem is completed.

From above theorems, it is observed that the nature of the fixed points of $g_{\lambda, n}(x)$ changes when parameter $\lambda$ crosses certain parameter value for both $n$ even or $n$ odd.

\section{Singular values of $g_{\lambda, n} \in \mathcal{G}$}

In this section, we describe the singular values of $g_{\lambda, n} \in \mathcal{G}$. The following theorem explains that the function $\mathrm{g}_{\lambda, \mathrm{n}} \in \mathcal{G}$ has infinitely many singular values.

Theorem 3.1. Let $\mathrm{g}_{\lambda, \mathrm{n}} \in \mathcal{G}$. Then, the function $\mathrm{g}_{\lambda, \mathrm{n}}(z)$ possesses infinitely many singular values.

Proof. For critical points of $g_{\lambda, n}(z), g_{\lambda, n}^{\prime}(z)=\lambda \frac{\left(b^{z}-1\right)^{n}-n z \ln b\left(b^{z}-1\right)^{n-1} b^{z}}{\left(b^{z}-1\right)^{2 n}}=0$. From this, we get the equation $(1-n z \ln b) b^{z}-1=0$. Using the real and imaginary parts, we have

$$
\begin{aligned}
& \frac{y \ln b}{\sin (y \ln b)}-\frac{1}{n} e^{y \ln b \cot (y \ln b)-\frac{1}{n}}=0, \\
& x=\frac{1}{n \ln b}-y \cot (y \ln b) .
\end{aligned}
$$

For $b>1$, it is seen that, from Figure 2 (a), equation (3.1) has infinitely many solutions for $n=3$ and $\mathrm{b}=5$ since number of intersections increases if interval increases along horizontal axis . For $0<\mathrm{b}<1$, similarly it is observed, from Figure 2 (b), equation (3.1) has infinitely many solutions for $n=3$ and $\mathrm{b}=0.5$.

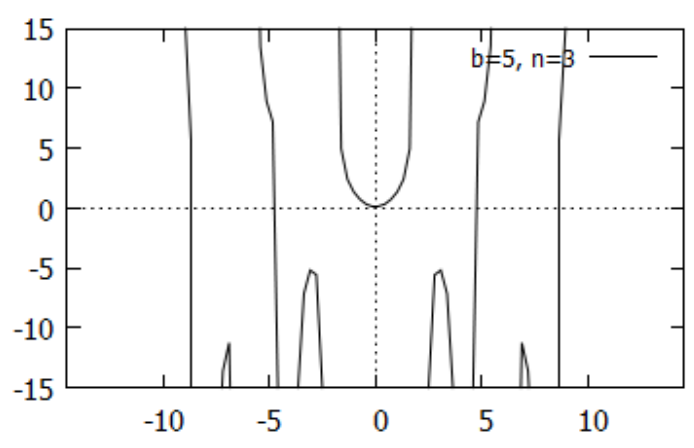

(a) For $n=3$ and $b=5$.

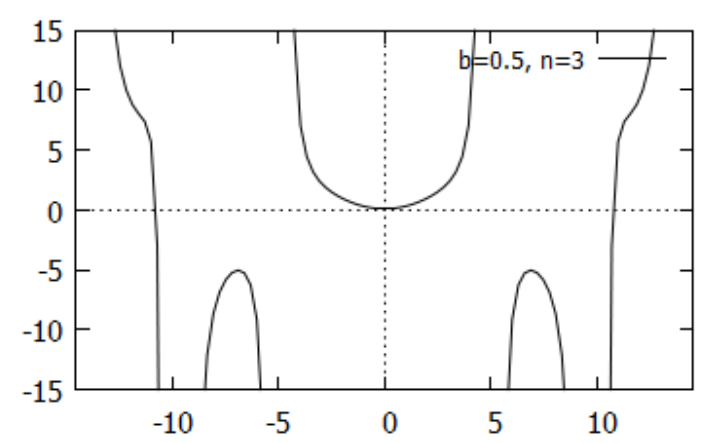

(b) For $n=3$ and $b=0.5$.

Figure 2: Graphs of $\frac{y \ln b}{\sin (y \ln b)}-\frac{1}{n} e^{y \ln b \cot (y \ln b)-\frac{1}{n}}$ (a) for $n=3$ and $b=5$, and (b) for $n=3$ and $b=0.5$.

Let $\left\{y_{k}\right\}_{k=-\infty, k \neq 0}^{k=\infty}$ be solutions of equation (3.1) for $b>1$. From equation (3.2), $x_{k}=\frac{1}{n \ln b}-$ $y_{k} \cot \left(y_{k} \ln b\right)$ for $k= \pm 1, \pm 2, \pm 3, \ldots$. Therefore, it follows that $z_{k}=x_{k}+i y_{k}$ are critical points of 
$g_{\lambda, n}(z)$ since $g_{\lambda}^{\prime}\left(z_{k}\right)=0$, and then the critical values are given by $g_{\lambda, n}\left(z_{k}\right)$. It is easily observed that $g_{\lambda, n}\left(z_{k}\right)=\lambda \frac{z_{k}}{\left(b^{2} k-1\right)^{n}}$ are distinct for different $k$. It shows that $g_{\lambda, n} \in \mathcal{G}$ has infinitely many critical values for $b>1$.

Similarly, we can obtain result for $0<\mathrm{b}<1$ using similar arguments as above.

The finite asymptotic value of $g_{\lambda, n}(z)$ is 0 since $g_{\lambda, n}(z) \rightarrow 0$ as $z \rightarrow \infty$ along the positive real axis for $\mathrm{b}>1$ and along the negative real axis for $0<\mathrm{b}<1$.

Thus, it proves that $g_{\lambda, n} \in \mathcal{G}$ possesses infinitely many singular values.

Remark 3.2. The rigorous theoretical calculation to show infinitely many solutions of equation (3.1) is not a very easy task. To achieve our goal, we use graphical utility to observe infinitely many solutions. One may be interested to obtain theoretical rigorous proof, they can construct it similar as [3, Proposition 1.2].

Let $\mathrm{H}^{+}=\{z \in \hat{\mathrm{C}}: \operatorname{Re}(z)>0\}$ be the right half plane and $\mathrm{H}^{-}=\{z \in \hat{\mathrm{C}}: \operatorname{Re}(z)<0\}$ be the left half plane. The following result shows that the function $g_{\lambda, n}^{\prime}(z)$ has no zeros in the right half plane for $b>1$ and no zeros in the left half plane for $0<\mathrm{b}<1$.

Theorem 3.3. Let $\mathrm{g}_{\lambda, \mathrm{n}} \in \mathcal{G}$. Then, the function $\mathrm{g}_{\lambda, \mathrm{n}}^{\prime}(\mathrm{z})$ has no zeros in (i) the right half plane $\mathrm{H}^{+}$for $\mathrm{b}>1$; (ii) the left half plane $\mathrm{H}^{-}$for $0<\mathrm{b}<1$.

Proof. Since $g_{\lambda, n}^{\prime}(z)=\lambda \frac{(1-n z \ln b) b^{z}-1}{\left(b^{z}-1\right)^{n+1}}=0$, then we have the equation $b^{-z}=1-n z \ln b$. It can be written as

$$
\frac{\cos (y \ln b)-i \sin (y \ln b)}{e^{x \ln b}}=1-n x \ln b-i n y \ln b .
$$

(i) For $b>1$, when $y \neq 0$, by imaginary part of equation (3.3), we have $\frac{\sin (y \ln b)}{y \ln b}=n e^{x \ln b}>1$ since $x>0$. This is not true for $y>0$. It is also false for $y<0$ because $\frac{\sin (y \ln b)}{y \ln b}$ is an even function.

When $y=0$, then $z=x>0$ and, by the real part of equation (3.3), $e^{x \ln b}=\frac{1}{1-n \times \ln b}$. For $x>\frac{1}{n \ln b}$ and $n \geqslant 2$, it is not valid because the left hand side is positive and the right hand side is negative. It is obviously not true when $x=\frac{1}{n \ln b}$. For $0<x<\frac{1}{n \ln b}$, define the function $q(x)=\frac{1}{1-n x \ln b}-e^{x \ln b}$. Now, we show that $q(x)>0$ for $0<x<\frac{1}{n \ln b}$. Using the series expansion, we have

$$
\begin{aligned}
q(x) & =1+n x \ln b+(n x \ln b)^{2}+(n x \ln b)^{3}+\cdots-\left(1+\frac{x \ln b}{1 !}+\frac{(x \ln b)^{2}}{2 !}+\frac{(x \ln b)^{3}}{3 !}+\cdots\right) \\
& =\left(n-\frac{1}{1 !}\right) x \ln b+\left(n^{2}-\frac{1}{2 !}\right)(x \ln b)^{2}+\left(n^{3}-\frac{1}{3 !}\right)(x \ln b)^{3}+\cdots .
\end{aligned}
$$

Since $n \geqslant 2, b>1$, and $x>0$, it follows that $q(x)>0$. Hence, there is no zeros for $0<x<\frac{1}{n \ln b}$. For instance, it can also be observed from Figure 3 that the function $q(x)$ is positive for $b=5$ and $\mathrm{n}=2,3,4,5, \ldots$

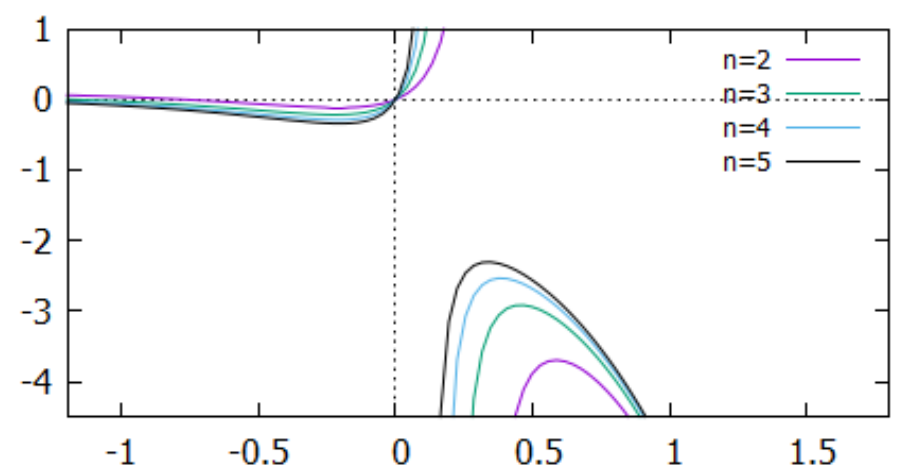

Figure 3: Graphs of $\frac{1}{1-n \times \ln b}-e^{x \ln b}$ for $b=5$ and $n=2,3,4,5$.

Therefore, it follows that the function $g_{\lambda, n}^{\prime}(z)$ has no zeros in $\mathrm{H}^{+}$for $b>1$. 


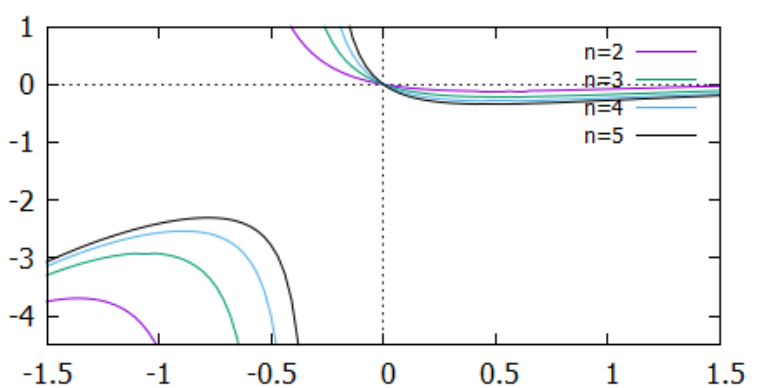

Figure 4: Graphs of $\frac{1}{1-n \times \ln b}-e^{x \ln b}$ for $b=0.5$ and $n=2,3,4,5$.

(ii) For $0<b<1$, similar as (i) for $b>1$, we can obtain the proof of this part (see Figure 4). Thus, the function $\mathrm{g}_{\lambda, \mathrm{n}}^{\prime}(z)$ has no zeros in $\mathrm{H}^{-}$for $0<\mathrm{b}<1$. This completes the proof.

Remark 3.4. For the zeros of $g_{\lambda, n}^{\prime}(z)$ on imaginary axis, from equation (3.3), we have $\cos (y \ln b)-i \sin (y \ln b)$ $=1-i n y \ln b$. This equation gives $y=0$. It follows that $g_{\lambda, n}^{\prime}(z)$ has no zeros on imaginary axis for $b>0$, $\mathrm{b} \neq 1$.

The following theorem shows that the function $g_{\lambda, n} \in \mathcal{G}$ maps the left half plane for $b>1$ and the right half plane for $0<\mathrm{b}<1$ in the closure and into the exterior of the disk according to two different regions, respectively.

Theorem 3.5. Let $\mathrm{g}_{\lambda, \mathrm{n}} \in \mathcal{G}$.

(a) For $\mathrm{b}>1$, the function $\mathrm{g}_{\lambda, \mathrm{n}}(\mathrm{z})$ maps $\mathrm{H}^{-}$

(i) in the closure of the disk centered at origin and having radius $\frac{|\lambda| \mathrm{R}}{(\ln \mathrm{b})^{n}}$ for $|z| \geqslant 1$ and $\mathrm{R}$ may be large.

(ii) into the exterior of the disk centered at origin and having radius $\frac{|\lambda|}{(\ln b)^{n}}$ for $|z|<1$.

(b) For $0<\mathrm{b}<1$, the function $\mathrm{g}_{\lambda, \mathrm{n}}(\mathrm{z})$ maps $\mathrm{H}^{+}$

(i) in the closure and into the exterior of the disk centered at origin and having radius $\left|\frac{\lambda R}{(\ln b)^{n}}\right|$ for $|z| \geqslant 1$ and $\mathrm{R}$ may be large.

(ii) into the exterior of the disk centered at origin and having radius $\left|\frac{\lambda}{(\ln b)^{n}}\right|$ for $|z|<1$.

Proof. Suppose the line segment $\gamma$ which is defined by $\gamma(t)=t z, t \in[0,1]$. Further, let us consider the function $\xi(z)=\mathrm{b}^{z}$ for an arbitrary fixed $z \in \mathbb{C}$. Then

$$
\int_{\gamma} \xi(z) d z=\int_{0}^{1} \xi(\gamma(t)) \gamma^{\prime}(t) d t=z \int_{0}^{1} b^{t z} d t=\frac{1}{\ln b}\left(b^{z}-1\right) .
$$

(a) For $\mathrm{b}>1$ and $z \in \mathrm{H}^{-}$,

$$
M_{1} \equiv \max _{\mathfrak{t} \in[0,1]}|\xi(\gamma(\mathrm{t}))|=\max _{\mathfrak{t} \in[0,1]}\left|\mathrm{b}^{\mathrm{t} z}\right|=\max _{\mathrm{t} \in[0,1]}\left|e^{\mathrm{tz} \ln \mathrm{b}}\right|<1
$$

and

$$
\mathrm{m}_{1} \equiv \min _{\mathrm{t} \in[0,1]}|\xi(\gamma(\mathrm{t}))|=\min _{\mathrm{t} \in[0,1]}\left|\mathrm{b}^{\mathrm{t} z}\right|=\min _{\mathrm{t} \in[0,1]}\left|e^{\mathrm{t} z \ln \mathrm{b}}\right|>\epsilon_{1}>0
$$

Then,

$$
\mathrm{m}_{1}|z| \ln \mathrm{b}<|z| \ln \mathrm{b} \leqslant\left|\mathrm{b}^{z}-1\right|=\left|\ln \mathrm{b} \int_{\gamma} \xi(z) \mathrm{d} z\right| \leqslant M_{1}|z| \ln \mathrm{b}<|z| \ln \mathrm{b} .
$$

For all $|z| \geqslant 1$ and $n \in \mathbb{N} \backslash\{1\}$, by the left side of inequality (3.4),

$$
\left|\frac{z}{\left(b^{z}-1\right)^{n}}\right| \leqslant \frac{1}{|z|^{n-1} m_{1}^{n}(\ln b)^{n}} \leqslant \frac{1}{m_{1}^{n}(\ln b)^{n}} .
$$


Assume $R=\frac{1}{m_{1}^{n}}$, we have

$$
\left|g_{\lambda, n}(z)\right|=\left|\lambda \frac{z}{\left(b^{z}-1\right)^{n}}\right| \leqslant \frac{|\lambda| R}{(\ln b)^{n}} .
$$

It shows that the function $\mathrm{g}_{\lambda, \mathrm{n}}(z)$ maps $\mathrm{H}^{-}$in the closure of the disk centered at origin and having radius $\frac{|\lambda| R}{(\ln \mathrm{b})^{n}}$ for all $|z| \geqslant 1$.

Similarly, using the right side of inequality (3.4),

$$
\left|\frac{z}{\left(b^{z}-1\right)^{n}}\right|>\frac{1}{|z|^{n-1}(\ln b)^{n}}>\frac{1}{(\ln b)^{n}} \quad \text { for all }|z|<1 \quad \text { and } \quad n \in \mathbb{N} \backslash\{1\} \text {. }
$$

Therefore, it follows that

$$
\left|g_{\lambda, n}(z)\right|=\left|\lambda \frac{z}{\left(b^{z}-1\right)^{n}}\right|>\frac{|\lambda|}{(\ln b)^{n}} \quad \text { for all } \quad|z|<1 .
$$

This gives that the function $\mathrm{g}_{\lambda, n}(z)$ maps $\mathrm{H}^{-}$into the exterior of the disk centered at origin and having radius $\frac{|\lambda|}{(\ln b)^{n}}$ for all $|z|<1$.

(b) For $0<\mathrm{b}<1$, and $z \in \mathrm{H}^{+}$,

$$
\mathrm{M}_{2} \equiv \max _{\mathrm{t} \in[0,1]}|\xi(\gamma(\mathrm{t}))|=\max _{\mathrm{t} \in[0,1]}\left|\mathrm{b}^{\mathrm{t} z}\right|=\max _{\mathrm{t} \in[0,1]}\left|\mathrm{e}^{\mathrm{tz} \ln \mathrm{b}}\right|<1
$$

and

$$
\mathrm{m}_{2} \equiv \min _{\mathrm{t} \in[0,1]}|\xi(\gamma(\mathrm{t}))|=\min _{\mathrm{t} \in[0,1]}\left|\mathrm{b}^{\mathrm{t} z}\right|=\min _{\mathrm{t} \in[0,1]}\left|e^{\mathrm{t} z \ln \mathrm{b}}\right|>\epsilon_{2}>0
$$

Then,

$$
\mathrm{m}_{2}|z||\ln \mathrm{b}|<|z||\ln \mathrm{b}| \leqslant\left|\mathrm{b}^{z}-1\right|=\left|\ln \mathrm{b} \int_{\gamma} \xi(z) \mathrm{d} z\right| \leqslant \mathrm{M}_{2}|z||\ln \mathrm{b}|<|z||\ln \mathrm{b}| .
$$

Now, similar as part (a), we can obtain the rest of the proof.

Thus, this completes the proof of theorem.

In the following theorem, it is proved that the function $g_{\lambda, n} \in \mathcal{G}$ has some critical values in the closure and other into the exterior of the disk centered at origin according to two different regions, respectively.

Theorem 3.6. Let $\mathrm{g}_{\lambda, \mathrm{n}} \in \mathcal{G}$. Then

(i) for $\mathrm{b}>1$, some critical values of $\mathrm{g}_{\lambda, \mathrm{n}}(\mathrm{z})$ lie in the closure of the disk centered at origin and having radius $\frac{|\lambda| \mathrm{R}}{(\ln \mathrm{b})^{n}}$ for $z \geqslant 1$ and other into the exterior of the disk centered at origin and having radius $\frac{|\lambda|}{(\ln \mathrm{b})^{n}}$ for $z<1$ in $\mathrm{H}^{-}$; and

(ii) for $0<\mathrm{b}<1$, some critical values of $\mathrm{g}_{\lambda, \mathrm{n}}(\mathrm{z})$ lie in the closure of the disk centered at origin and having radius $\left|\frac{\lambda R}{(\ln b)^{n}}\right|$ for $z \geqslant 1$ and other into the exterior of the disk centered at origin and having radius $\left|\frac{\lambda}{(\ln b)^{n}}\right|$ for $z<1$ in $\mathrm{H}^{+}$.

Proof.

(i) For $b>1$, by Theorem 3.3 (a), all the critical points of $g_{\lambda, n}(z)$ lie in $H^{-}$since $g_{\lambda, n}^{\prime}(z)$ has no zeros in $\mathrm{H}^{+}$. By Theorem 3.5 (a), the function $\mathrm{g}_{\lambda, n}(z)$ maps the left half plane $\mathrm{H}^{-}$in the closure of the disk centered at origin and having radius $\frac{|\lambda| R}{(\ln b)^{n}}$ for $|z| \geqslant 1$ and into the exterior of the disk centered at origin and having radius $\frac{|\lambda|}{(\ln b)^{n}}$ for $|z|<1$. Therefore, it follows that some critical values of $g_{\lambda, n}(z)$ lie in the closure of the disk centered at origin and having radius $\frac{|\lambda| R}{(\ln b)^{n}}$ for $|z| \geqslant 1$ and other into the exterior of the disk centered at origin and having radius $\frac{|\lambda|}{(\ln b)^{n}}$ for $z<1$ in $\mathrm{H}^{-}$.

(ii) For $0<\mathrm{b}<1$, we can proof this part similar as above. 


\section{Conclusions}

The real fixed point and singular values of a family of functions $\frac{z}{\left(\mathrm{~b}^{z}-1\right)^{n}}$ have been investigated here, where functions have arose from generating function of the unified generalized Apostol type polynomials. It is also a generalization of a family of functions $\frac{z}{\left(e^{z}-1\right)^{n}}$ on base $b$. The real fixed points of $g_{\lambda, n}(z)$ have been found with their nature and it is observed that these real fixed points have different nature for $n$ odd and $n$ even. We have shown that $g_{\lambda, n}(z)$ has infinitely many singular values for $n \in \mathbb{N} \backslash\{1\}$. We have also seen that some critical values of $g_{\lambda, n}(z)$ lie in the closure and other into the exterior of the open disk with center at origin according to two different regions in the left half plane and in the right half plane for $b>1$ and $0<b<1$, respectively.

\section{Acknowledgment}

The author is thankful to referees for providing helpful suggestions to improve this paper.

\section{References}

[1] T. K. Chakra,T. Nayak, Iteration of the translated tangent, Bull. Malays. Math. Sci. Soc., 2017 (2017), 16 pages. 1

[2] R. L. Devaney, Se $e^{x}$ dynamics, topology, and bifurcations of complex exponentials, Topology Appl., 110 (2001), $133-161$.

[3] G. P. Kapoor, M. G. P. Prasad, Dynamics of $\left(e^{z}-1\right) / z$ : the Julia set and bifurcation, Ergodic Theory Dynam. Systems, 18 (1998), 1363-1383. 1, 3.2

[4] M. G. Lee, C. C. Ho, Fixed points of two-parameter family of function $\lambda\left(\frac{x}{\mathrm{~b}^{x}-1}\right)^{n}$, Appl. Math., 6 (2015), 576-584. 1

[5] T. Nayak, M. G. P. Prasad, Julia sets of Joukowski-Exponential maps, Complex Anal. Oper. Theory, 8 (2014), 10611076. 1

[6] A. G. Radwan, On some generalized discrete logistic maps, J. Adv. Res., 4 (2013), 163-171. 1

[7] P. J. Rippon, G. M. Stallard, Transcendental Dynamics and Complex Analysis, Cambridge University Press, Cambridge, (2008). 1

[8] M. Sajid, On fixed points of one parameter family of function $\frac{x}{b^{x}-1}$ II, Int. J. Math. Anal. (Ruse), 8 (2014), 891-894. 1

[9] M. Sajid, On real fixed points of one parameter family of function $\frac{x}{b^{x}-1}$, Tamkang J. Math., 46 (2015), 61-65. 1

[10] M. Sajid, Singular Values of One Parameter Family $\lambda \frac{\mathrm{b}^{z}-1}{z}$, J. Math. Comput. Sci., 15 (2015), 204-208. 1

[11] M. Sajid, Singular values of one parameter family of generalized generating function of Bernoulli's numbers, Appl. Math. Inf. Sci., 9 (2015), 2921-2924. 1

[12] M. Sajid, Real fixed points and dynamics of one parameter family of function $\left(b^{x}-1\right) / x$, J. Association Arab Uni. Basic Appl. Sci., 21 (2016), 92-95. 1

[13] M. Sajid, Real fixed points and singular values of two-parameter family $\lambda z /\left(e^{z}-1\right)^{n}$, New Trends Math. Sci., 5 (2016),

[14] M. Sajid, Singular values of two parameter families $\lambda\left(\frac{\mathrm{b}^{z}-1}{z}\right)^{\mu}$ and $\lambda\left(\frac{z}{\mathrm{~b}^{z}-1}\right)^{\eta}$, J. Taibah Uni. Sci., 11 (2017), 324-327. 1

[15] M. Sajid, Bifurcation and chaos in real dynamics of a two-parameter family arising from generating function of generalized Apostol-type polynomials, Math. Comput. Appl., 23 (2018), 11 pages. 1

[16] M. Sajid, G. P. Kapoor, Chaos in dynamics of a family of transcendental meromorphic functions, J. Nonlinear Anal. Appl., 2017 (2017), 11 pages. 1

[17] S. Sharifi, M. Salimi, S. Siegmund, T. Lotfi, A new class of optimal four-point methods with convergence order 16 for solving nonlinear equations, Math. Comput. Simulation, 119 (2016), 69-90. 1

[18] H. M. Srivastava, Some generalizations and basic (or q-) extensions of the Bernoulli, Euler and Genocchi polynomials, Appl. Math. Inf. Sci., 5 (2011), 390-444. 1

[19] J. H. Zheng, On fixed-points and singular values of transcendental meromorphic functions Sci. China Math., 53 (2010), 887-894. 1 